\title{
Predicting Joint Effects on CubeSats to Enhance Internet of Things in GCC Region Using Artificial Neural Network
}

\author{
Faris A. Almalki $\mathbb{D D}^{1}$ and Soufiene Ben Othman $\mathbb{D}^{2}$ \\ ${ }^{1}$ Department of Computer Engineering, College of Computers and Information Technology, Taif University, P.O. Box 11099, \\ Taif 21944, Saudi Arabia \\ ${ }^{2}$ Prince Laboratory Research, IsitCom, University of Sousse, Hammam Sousse, Tunisia \\ Correspondence should be addressed to Soufiene Ben Othman; ben_oth_soufiene@yahoo.fr
}

Received 4 September 2021; Revised 28 October 2021; Accepted 5 November 2021; Published 25 November 2021

Academic Editor: Han Wang

Copyright (c) 2021 Faris A. Almalki and Soufiene Ben Othman. This is an open access article distributed under the Creative Commons Attribution License, which permits unrestricted use, distribution, and reproduction in any medium, provided the original work is properly cited.

\begin{abstract}
Satellite telecommunication systems promise to bridge digital gaps and deliver wireless communication services to any corner of the world. However, despite satellites' global connectivity and wide footprint, still atmospheric and dust impairments are open challenges that face satellite systems, especially at high-frequency bands in arid and semiarid regions. Therefore, this paper aims to predict joint effects of atmospheric and dust attenuations in Gulf Cooperation Council (GCC) countries on CubeSat communications using Artificial Neural Network (ANN). The prediction model has been carried out using a massive Multiple-Input Multiple-Output (MIMO) antenna payload at K-frequency Bands. Consider these joint effects have positive relations in calculating satellites link margin, which leads to obtaining efficient communication system, delivering better quality of service (QoS), and enhancing Internet of Things (IoT) connectivity, or even Internet of Space Things (IoST). Predicated results infer that the ANN attenuation predictions, along with the $5 \mathrm{G}$ MIMO antenna on-board the CubeSat, offer much promise channel model for satellite communications, which in turn leads to not only supporting IoT connectivity but also reducing power consumption, thus enhancing lifetime of CubeSat. Also, this study can provide a reference for CubeSat engineers to guarantee large-capacity communication.
\end{abstract}

\section{Introduction}

Traditional wireless communication systems provide services with a good level of data rates, reconfigurable provision with various dynamic coverage demands. However, the deployment of these enabling technologies has led to a huge rise in the demand for mobile communications, partly due to the exponential growth in multimedia traffic and the emergence of a new type of technology such as the Internet of Things (IoT) or Big Data. However, space-based wireless communication systems such as satellites and unmanned aerial vehicles (UAVs) promise to provide global connectivity, because they contribute effectively to linking trillions of objects and sensors, all of which generate real-time data. This also helps in boosting IoT and Internet of Everything (IoE) technologies, which are considered fuel for the Fourth Industrial Revolution (4IR) [1-3]. Figure 1 shows the main technological pillars of 4IR include IoT and space-based vehicles (e.g., Drones and Satellites). The 4IR and its enormous processing power represent creative digitization based on a combination of symbiotically interacting technological breakthroughs through innovative algorithms. Further, the combination between IoT and Drones and Satellites is vital, which would massively pave the way to hyperconnected societies, thus leading to global smart connectivity [4-6].

Figure 1 displays that Artificial Intelligent (AI) is one of the key pillars of 4IR, where such an innovative technology can be considered a great booster of the 4IR and digital transformation in any modern society. One of those AI techniques is an Artificial Neural Network (ANN), which is a computing system inspired by the biological neural networks that constitute biological brains, where all neurons contain individual weights and bias values that are parallel and organized nonlinear components. Further, the ANN technique 


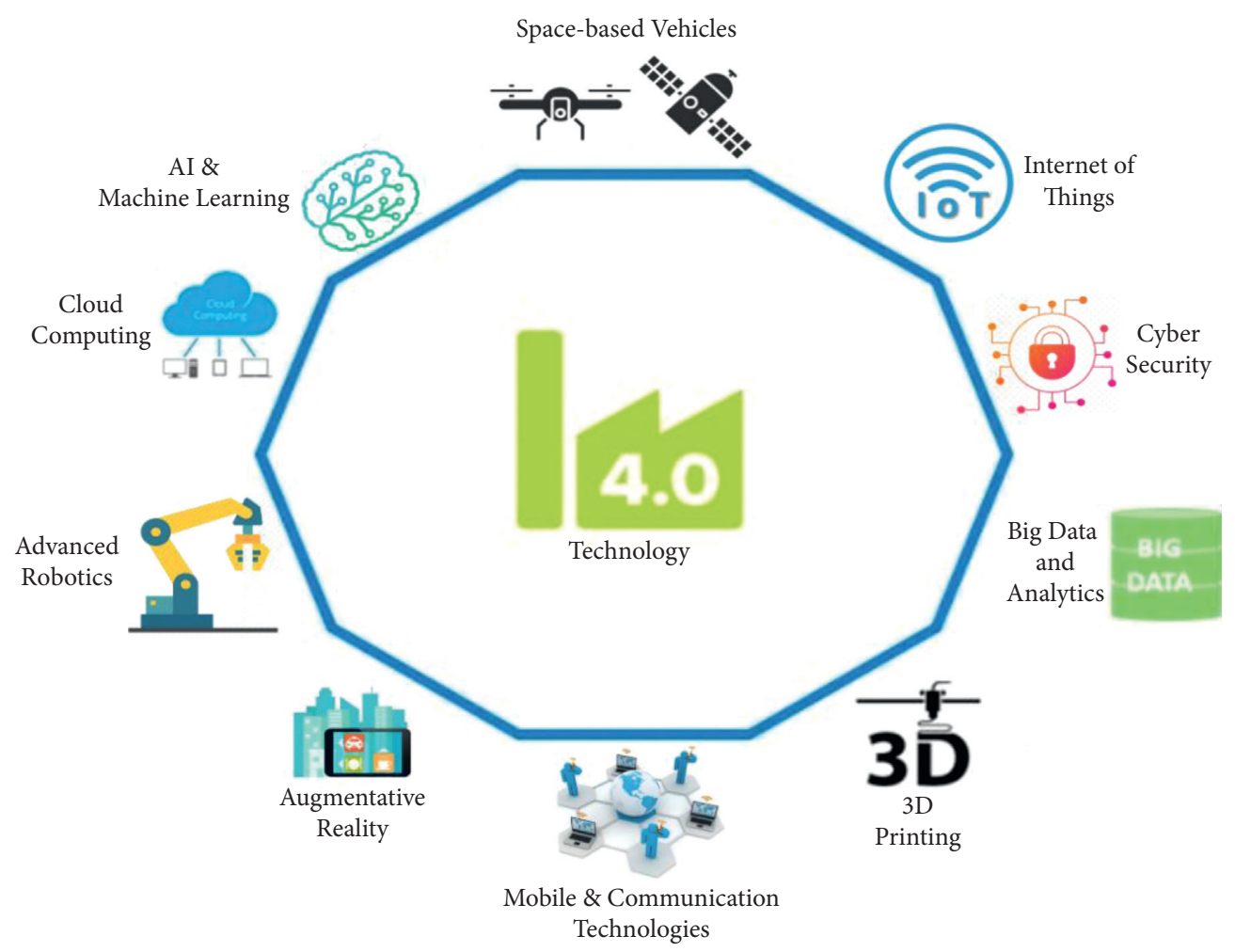

FIgURE 1: The main 4IR pillars.

is widely used for highly accurate predictions and/or optimization due to its simplicity and efficiency [7]. The authors in [8-11] are representative examples of related work that considered ANN for optimization and enhancement.

Researchers in space-based systems confirm that reliability, flexibility, efficiency, wide applicability, Line of Sight (LoS) connectivity, low latency, rapid deployment, and costeffectiveness are the main advantages of using such a technology. The authors have emphasised that space is stepping up to the connectivity challenge posed by the 4IR, where one of the driving forces of this change has been the introduction of next-generation high-technology cube satellite systems [12-14]. Further, space-based communication systems include satellites enticing due to their various applications and low design-and-deployment cost [15-17]. Furthermore, in the last few years, there has been a tremendous interest in cube satellites, which are also known as "CubeSats" or "Nanosatellites," built to standard dimensions of few tens on centimetres and weigh few kilograms. These small satellites can fly on a rocket to space to provide opportunities for small satellite payloads to serve some applications as follows $[18,19]$ :

(i) Technology demonstration: in the harsh environment of space to test new instruments or materials

(ii) Academia and educational projects: which provide a unique hands-on experience in developing space missions from design to launch and operations

(iii) Commercial applications: telecommunications, remote sensing, high-resolution aerial imagery, ship tracking, and formation flying
The nanosatellites that operate at Low Earth Orbit (LEO) based on Van Allen radiation belts at altitude start from around $160 \mathrm{~km}$ to $600 \mathrm{~km}$ from the Earth facing some challenges, for instance, propagation impairments in last-mile communications, especially when using high-frequency bands often assigned to space-based communication systems (e.g., above $10 \mathrm{GHz}$ ). Although high-frequency bands offer wide bandwidths, they are more vulnerable to signal degradation, as radio signals get absorbed by atmospheric rain, snow, ice, or even dust. The attenuations' factors vary from one region to another worldwide [20-22]. The authors in [23, 24] introduced a holistic overview of various aspects of CubeSats missions, services, recent advances, and future challenges for further research. The authors stressed that the research on cube satellites for communications is still in its early phase. IoT, Internet of Space Things (IoST), low-power long-range networks, Iridium communications, earth-remote sensing, and machine learning are opened research areas for cube satellites. The authors in [25] have introduced CubeSats as "Eyes in the Sky" because it monitors and offers various remote sensing with capabilities of IoST. According to a forecast by SpaceWorks Enterprises, Inc. (SEI), more than 2,800 nano-/microsatellites will require launch over the next five years. Figure 2 shows a comparison between small satellites application trends that weigh $(1-50 \mathrm{~kg})$, where the left pie chart shows application trends from 2014 to 2018, while the right one from 2019 to 2023 [26]. Figure 3 illustrates Cube satellite wide applications.

Woellert et al. [27] shed light on CubeSats capabilities in science education and spur technology for developing nations. Aslan et al. [28] constructed a CubeSat project in Sharjah-Emirates, one of the Gulf Cooperation Council 


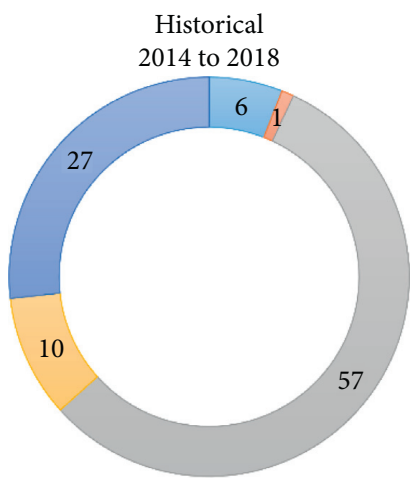

- Communications

$\square$ Scientific

$\square$ Noval Applications

$\square$ Technology

$\square$ Earth Observation

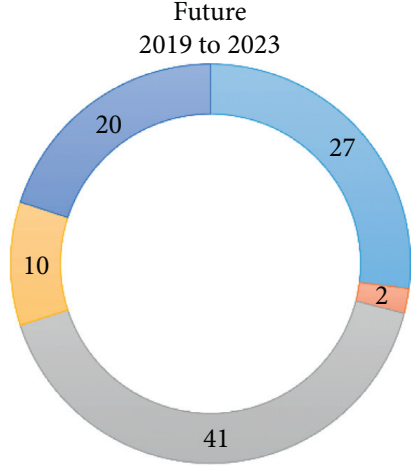

- Communications

$\square$ Scientific

$\square$ Noval Applications

$\square$ Technology

$\square$ Earth Observation

Figure 2: Cube satellite application trends $(1-50 \mathrm{~kg})$.

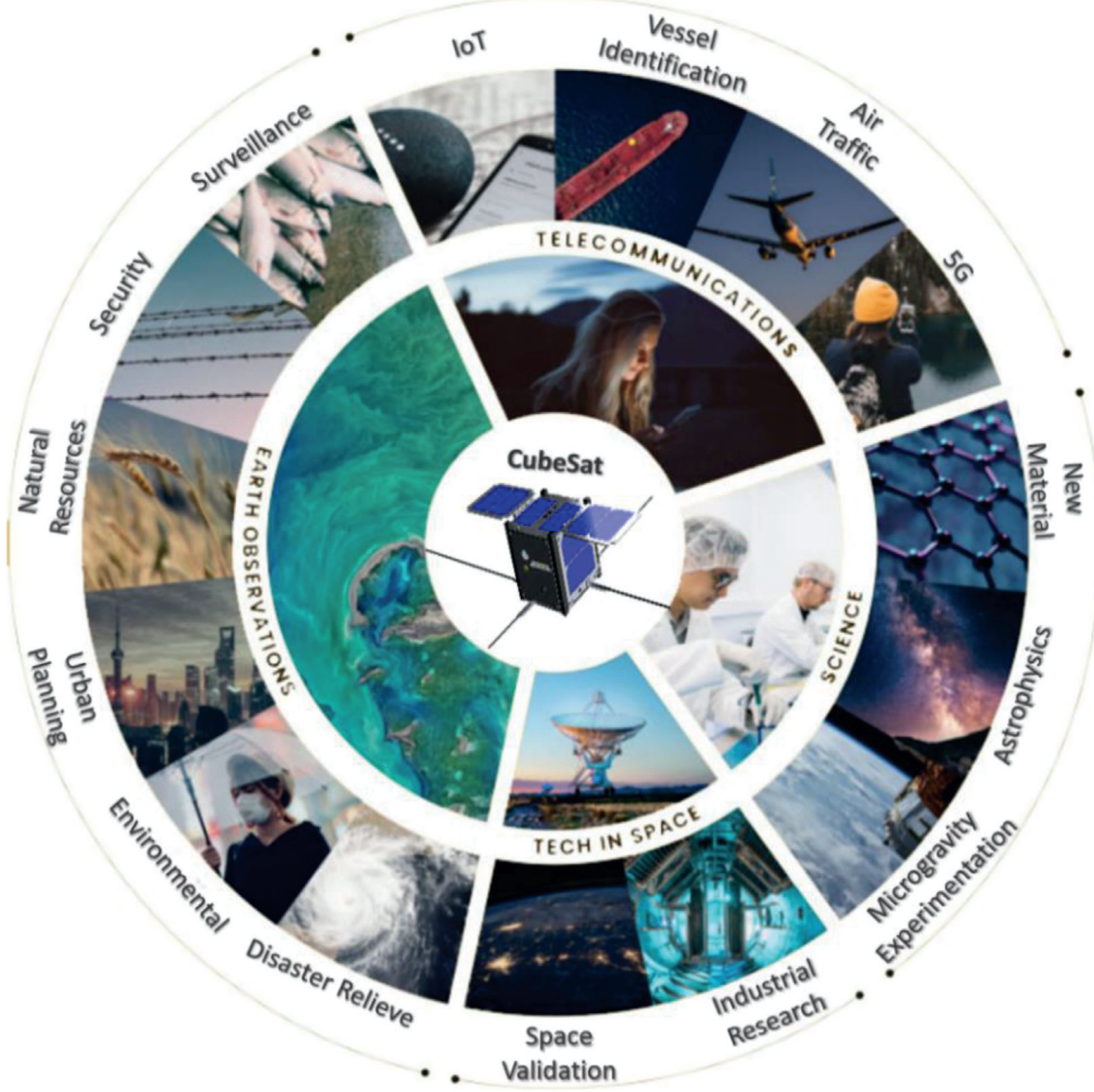

FIGURE 3: Cube satellite wide applications.

(GCC) countries. The project aims to educate students in cutting edge technologies including space technology, which puts human capacity development in its centre and support United Nations Sustainable Development Goals (UN SDG) for 2030. Akyildiz et al. [29] highlighted the importance of atmospheric attenuation when designing link budget analysis at an altitude of $400 \mathrm{~km}$ with above $10 \mathrm{GHz}$ frequency.
$\mathrm{Qu}$ et al. [30] focused on rain attenuation caused by satellite communication operating on frequency bands above $10 \mathrm{GHz}$, as they are sensitive to atmosphere physical phenomena. The work predicted real-time satellite channel fading caused by rain using stochastic dynamic modelling (SDM). Luini and Capsoni [31] addressed the joint effects of rain and clouds on Ka-Band for LEO satellite using 
stochastic model. The results give better understanding of link budget; however, more atmospheric effects (e.g., gasses, water vapor, and oxygen) are recommended for further work.

Mpoporo and Owolawi [32] inferred that rain attenuation is the most unfavourable impact on signal transmission at high-frequency bands, especially in places with high rainfall. This paper aims to estimate rain attenuation on satellite links using the ITU-R model in South Africa. Further, Wang et al. [33] gave rain attenuation analysis of the Ka-Band satellite communication in the Indian and Pacific Oceans. Reasonable results of the ITU-R model in predicting rain attenuation have been presented. Averly and Suryana [34] also considered the ITU-R model and simulated it in MATLAB at below $3 \mathrm{GHz}$ frequencies. However, Regonesi et al. [35] emphasised that the ITU-R model with some limitations and inaccuracies, especially in high frequencies elevation angles and rainfall cases.

An experimental work of radio link design for a CubeSat communication system is presented in [36]. The CubeSat is placed at $400 \mathrm{~km}$ altitude using a dipole antenna with an omnidirectional pattern and frequency band of $401 \mathrm{MHz}$. Experimental results of link budget are $137 \mathrm{~dB}$ of free space path loss (FSPL), $5 \mathrm{~Kb} / \mathrm{S}$ of effective throughput, and signalto-noise ratio (SNR) floats between $10 \mathrm{~dB}$ and $24.54 \mathrm{~dB}$ at bit error rate (BER) of $1 \times 10^{-5}$. Atmospheric effects have been neglected.

Charbit et al. [37] showed a Narrow-Band IoT (NB-IoT) for satellite-to-ground communication with atmospheric effects. Carrier-to-noise ratio (CNR) of satellite links are evaluated at altitude of $600 \mathrm{~km}$ and $2 \mathrm{Ghz}$ frequency band with wide consideration of atmospheric effects (gasses, rain, scintillation). Results are adequate, but the work does not include high-frequency bands, where it truly shows the predictions of the atmospheric effects.

Harb et al. [38] confirmed that dust is a vital cause of wireless channel impairments, which are regularly observed in arid and semiarid areas worldwide. References [38, 39] presented a dust storm model that affects high-frequency satellite-to-ground communications in Saudi Arabia and other countries in the Middle East. The dust model includes mathematical variables such as visibility, average dust particles size, and permittivity indices. SNR of various elevation angles, altitude, and frequency bands have been analysed in case of using a directional-hemispherical antenna. Predications of dust attenuations help in optimally designing satellite link budgets.

Motivated by the below observations through the related studies, major portions of satellite link budgets have considered either part of atmospheric impairments mainly rains attenuation or dust attenuation separately. Therefore, this paper aims to provide a comprehensive prediction model that encompasses joint effects of atmospheric and dust impairments from CubeSat communication aspect in the GCC region using ANN. The prediction model has been carried out using a massive Multiple-Input Multiple-Output (MIMO) antenna payload at K-frequency Bands. The massive MIMO has combined with Orthogonal Frequency Division Multiplexing (OFDM), which can divide a radio channel into many close-spaced subchannels to deliver extra reliable communications over multiple antennas at high speeds. The atmospheric effects comprise rain, gasses, clouds, scintillation, and dust. Figure 4 shows a bird's eye view of a cube satellite network communication architecture, which consists of space segment and ground segment. The former has the cube satellite with communication payloads at the LEO layer. The latter shows a ground station and many wireless communication links within a footprint that can serve fixed/mobile users. Clearly, signals get attenuated as they pass by atmospheric effects, affecting the received signals at the ground users. Services such as Internet connectivity and active or passive sensing can benefit from the strong received signal from the CubeSat side.

The rest of this paper is organized as follows: Section 2 presents the proposed predictions model followed by Section 3 that details the performance analysis and discusses results. Section 4 concludes this paper.

\section{Proposed Predictions Model}

Predicating weather impairments is an essential component when evaluating optimal link budgets for satellite communication links. This section sheds light on the proposed prediction model that comprehensively includes joint effects of atmospheric and dust impairments from CubeSat to GCC countries using an ANN technique. Since researchers and designers need to consider a wide range of parameters that can impact communication links. This work considers a CubeSat simulation library for Aerospace Blockset in MATLAB. It simulates, analyses, and visualizes the motion and dynamics of CubeSats and nanosatellites, as Figure 5 shows the Cube satellite network communication architecture. The CubeSat simulation toolbox provides standardsbased tools and enormous parameters and network configurations for designing, simulating, and verifying satellite communications systems and links. This section contains three subsections: (a) CubeSat Link Margin, (b) Atmospheric Effects Calculation, and (c) ANN Framework.

2.1. CubeSat Link Margin. Calculating atmospheric and dust impairments is affecting the total carrier-to-noise ratio $(\mathrm{C} /$ $\mathrm{N})$, as per equations (1) and (2), because it is one of the performance quality indicators of a satellite communication channel. Further, the most critical performance parameter is bit energy per noise density ratio $\left(E_{b} / N_{0}\right)$ as per equation (3), which is also influenced over a satellite network due to its characteristics such as long propagation, frequency, and bandwidth $[40,41]$ :

$$
\begin{aligned}
\left(\frac{C}{N}\right)_{T}= & P_{t}+G_{C}+G_{S}-\left(\frac{4 \pi d}{\lambda^{2}}\right) \\
& -F-10 \log _{10}\left(K T_{0} B\right)-\text { Losses, } \\
\mathrm{d}[\mathrm{km}]= & 2 E_{r}\left[\cos ^{-1}\left(\frac{E_{r}}{E_{r}+h_{t}} * \cos (\theta)\right)-\theta\right],
\end{aligned}
$$




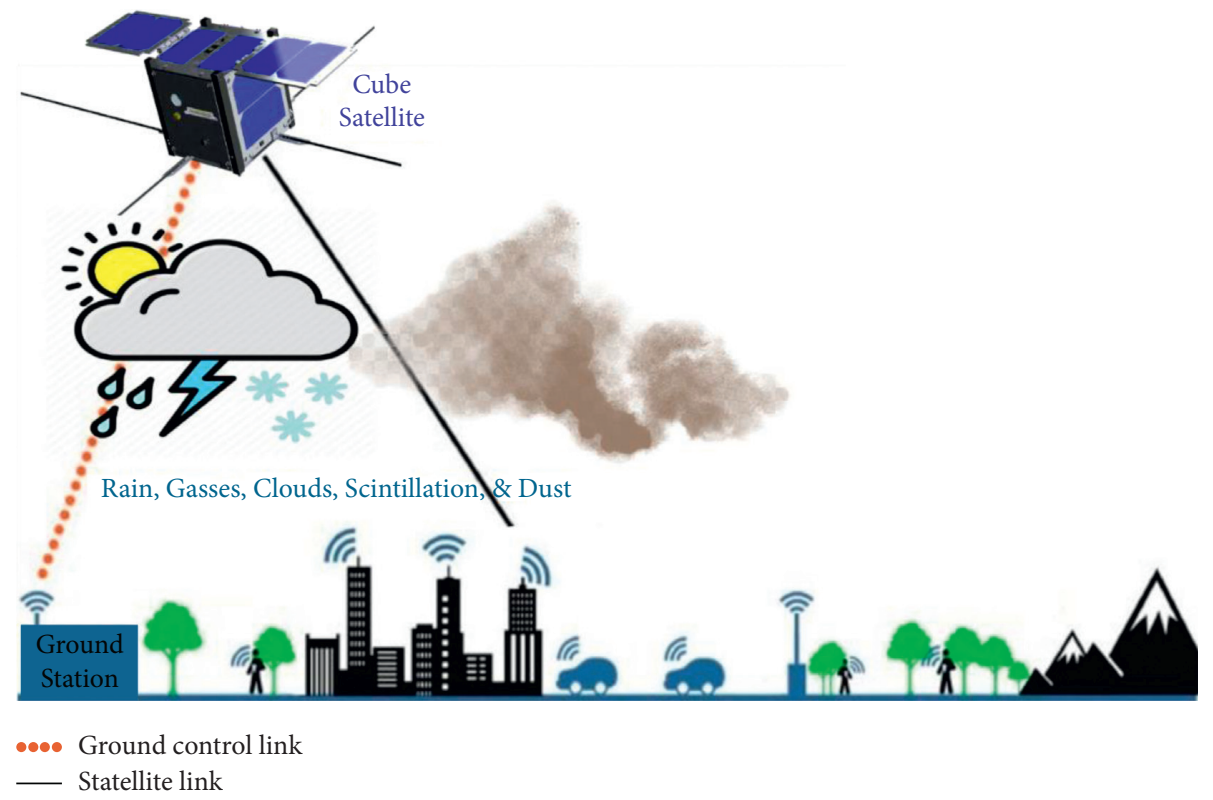

FIgURE 4: Cube satellite network communication architecture.

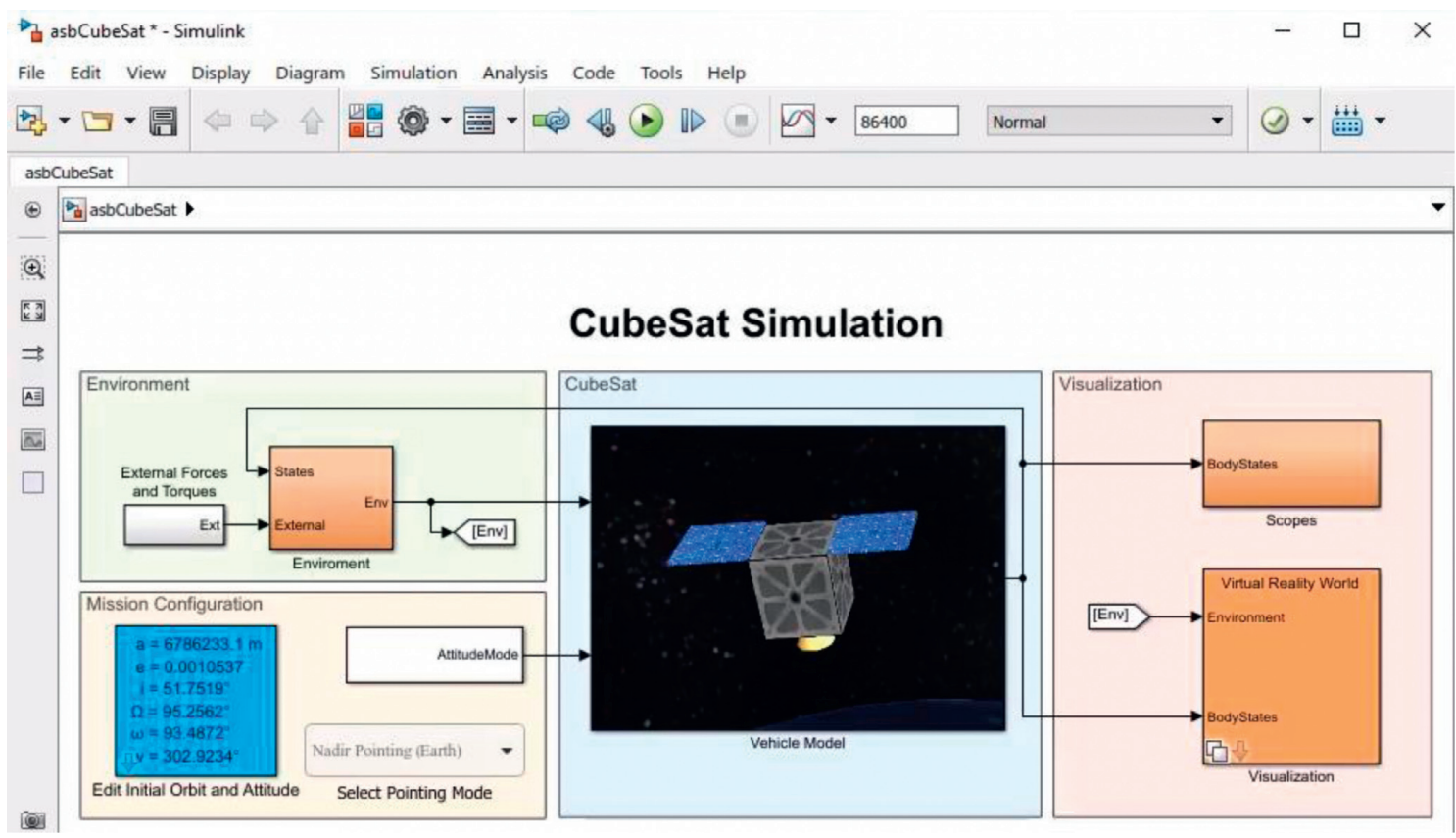

Figure 5: CubeSat network communication architecture.

$$
\frac{E_{b}}{N_{0}}=\left(\frac{C}{N}\right)_{T} \cdot\left(\frac{B w}{R_{b}}\right),
$$

where $P_{t}$ denotes transmitter power; $G_{C}$ denotes CubeSat antenna gain; $G_{S}$ denotes ground station antenna gain; $d$ denotes the distance from CubeSat to Earth station/terrestrial users; $\lambda$ denotes wavelength, which is the ratio of the speed of light (c) to the frequency of transmission $f$; $d$ is calculated with consideration of elevation angle $\theta$, which is a vital aspect in space-based wireless communication systems to achieve better LoS connectivity; $E_{r}$ denotes the Earth's radius $(6,371 \mathrm{~km}) ; h_{\mathrm{t}}$ denotes CubeSat's altitude; $F$ denotes the receiver noise figure; $K$ denotes Boltzmann's constant (1.38.10-23 Ws/K); $T_{0}$ denotes the absolute temperature (290K); B denotes the receiver noise bandwidth; Losses represent atmospheric (rain, gasses, clouds, and scintillation) and dust attenuations; $B w$ denotes the required bandwidth; and $R_{b}$ denotes data bit rate.

A massive 5G MIMO antenna is used in the proposed model design, where it consists of a planar phased array of 64 patches designed for operation at up to tens of $\mathrm{GHz}$, along with an optimum feed location to produce optimal return 
loss. Further, the considered massive 5G MIMO antenna uses adaptive beamforming with spatial multiplexing to increase data rates and decrease interference. To propagate signals well from CubeSat to Earth stations/users, each patch of massive 5G MIMO antenna uses an adaptable phase offset to steer beamforming at various angles $\left({ }^{\circ}\right)$ towards the desired direction, where the main beams can be calculated as follows [42-44]:

$$
W_{n}=\exp \left[-j\left(\frac{2 \pi}{\lambda}\right) \sin \left(\theta_{d}\right)\left[\begin{array}{ll}
x_{n} & \left.\left.\cos \left(\phi_{d}\right)+y_{n} \sin \left(\phi_{d}\right)\right]\right]
\end{array}\right.\right.
$$

where $\theta_{d}, \phi_{d}$ denote phases at $x_{n}$ and $y_{n}$.

2.2. Atmospheric Effects Calculation. This section presents the mathematical modelling used to evaluate the performance of CubeSat links and determine different types of atmospheric losses. These atmospheric and dust impairments can seriously affect satellite-to-Earth links at high frequencies, which are often above $10 \mathrm{GHz}$. Equations (5)-(9) represent losses (attenuations) of rain, gasses, clouds, scintillation, and dust, respectively $[33,38,39,45,46]$ :

$$
\begin{aligned}
R_{A}= & \alpha R^{K_{\prime}} \cdot h_{t}, \\
G_{A}= & \frac{A_{0}+A_{w}}{\sin \theta}, \\
C_{A}= & \gamma_{\text {cloud }}\left(\frac{\mathrm{LWC}}{\sin \theta}\right), \\
S_{A}= & a(p) \cdot \sigma, \\
D_{A}= & \frac{\left(5.67 \times 10^{2}\right)}{V(\theta) \cdot r_{e 0}^{2} \cdot \lambda} \\
& * \frac{\varepsilon^{\prime \prime}}{(\varepsilon l+2)^{2}+\varepsilon^{\prime \prime 2}} \sum_{i}^{n} p_{i} * r_{i}^{3},
\end{aligned}
$$

where $R_{A}$ denotes rain attenuation, $\alpha$ and $K I$ are empirical coefficients that depend on frequency band and temperature, $R$ denotes rain rate in $\mathrm{mm} / \mathrm{hr}, G_{A}$ denotes gasses attenuation, denotes oxygen absorption, $A_{w}$ denotes water vapor, $C_{A}$ denotes cloud attenuation, LWC denotes liquid water content, $S_{A}$ denotes scintillation attenuation, $a(p)$ denotes the time percentage factor for time percentage, $\sigma$ denotes standard deviation of the signal amplitude, $D_{A}$ denotes dust attenuation as a function of propagation angle and frequency, $V$ denotes visibility in $\mathrm{Km}, r_{e 0}^{2}$ denotes equivalent particle radius, $\varepsilon$ /and $\varepsilon^{\prime \prime}$ are real and imaginary parts of the dielectric constant of dust, and $\sum_{i}^{n} p_{i} * r_{i}^{3}$ denote summation of the probability particle size between $r_{i}$ series of particle volume at the lowest and highest layer level.

2.3. ANN Framework. There are various types of machine learning techniques, which are widely used for high accurate predictions/optimization. One of those well-known techniques is ANN that mainly comprises three layers: input layer, hidden layer, and output layer. Figure 6 shows universal illustration of an ANN, where all neurons contain individual weights and bias values that are parallel and organized nonlinear components, which are repeatedly appropriate for improving performance during training [47-51].

This work uses Multilayer Feedforward Network Architecture (MLP), which is one of the most common type of ANNs used in the literature. MLP is used to predict the link margin of CubeSat as per the following equations:

$$
\begin{aligned}
\alpha & =b+\sum_{n=1}^{N} y_{n} t_{n}, \quad n=1,2, \ldots, N, \\
\chi(\alpha) & =\frac{1}{\left(1+e^{-\alpha}\right)}, \\
E & =\frac{1}{2} \sum_{h-1}^{H}\left(A_{0}-D_{0}\right)^{2}\left(\frac{m m}{h}\right), \\
\frac{\partial E_{\text {total }}}{\partial A_{0}} & =-\left(D_{0}-A_{0}\right), \\
\Delta w_{i} & =\eta \frac{\partial E}{\partial A_{i}}, \quad i=1,2, \ldots, I,
\end{aligned}
$$

where $\alpha$ denotes the additive and output function, which is a sigmoid activation function of $\chi$ that denotes neuron prediction, $b$ denotes bias input, $y_{n}$ denotes nth weight, $t_{n}$ denotes nth input, and $\mathrm{N}$ denotes the number of inputs. $E$ estimates the error when training the ANN occurs, $D_{0}$ denotes expected output (target), $\mathrm{mm}$ denotes multilayer of the feedforward network, $A_{0}$ denotes real output, $H$ denotes the data point number, $w$ denotes optimal weight vector, $\Delta w_{i}$ denotes the change in weight on the ith input, $\eta$ denotes learning rate, and $i$ denotes the input, and $I$ denotes the last input of the neural network. The training algorithm stops when the size of the error function is minimal enough.

Figure 7 demonstrates the proposed ANN framework to predict the link margin of CubeSat with consideration of atmospheric and dust impairments. Such an ANN framework seems massive parallel architectures, yet it operates with interconnected mechanisms efficiently to solve difficult problems. A Levenberg-Marquardt backpropagation algorithm is used in the feedback forward fitting tool to evaluate the performance of the ANN predictions, where the Levenberg-Marquardt algorithm uses the Hessian matrix approximation, as per equation (15), where it is considered faster and more accurate near an error minimum. Hence, the scalar $\mu$ decreases after each drop-in performance function, which indicates the performance function is always reduced at each iteration of the algorithm [52-56]:

$$
x k+1=x k-[J T J+\mu J]-1 J T_{e},
$$

where $J$ denotes a Jacobian matrix, which consists of the first values of the network errors considering the assigned weights and biases and $e$ denotes a vector of network errors. Input vectors and target vectors are arbitrarily divided into three sets: 


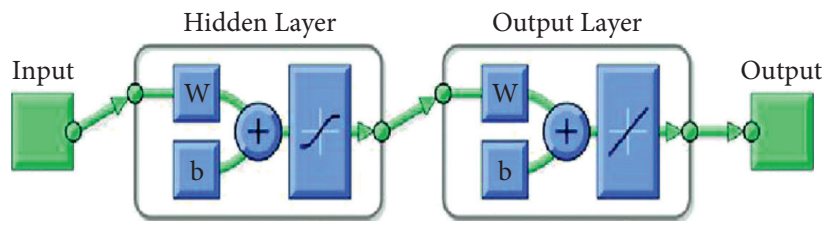

Figure 6: ANN's three universal illustration layers.

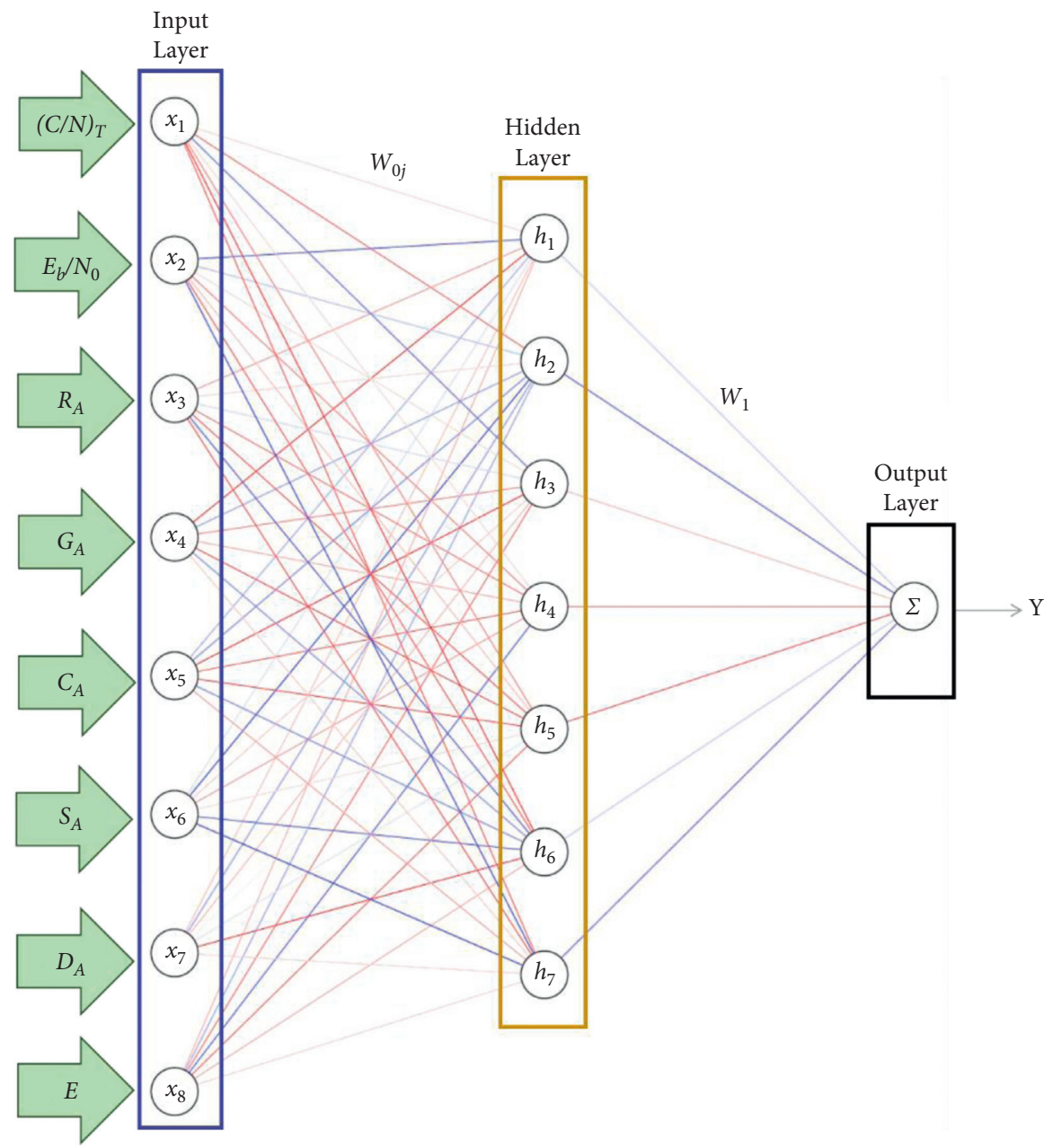

FIgURE 7: The proposed ANN framework.

(1) Training makes offerings to the ANN while training, and the network is tuned in response to its error, consequently calculating the gradient and updating the weights and biases.

(2) Validation measures network generalization and stops training when generalization stops improving.

(3) Testing delivers an autonomous performance measure during and after training, hence with no effect on training.

\section{Performance Analysis and Discussions}

Predictions of joint effects of atmospheric and dust attenuations for CubeSat communications associated with a massive MIMO antenna in GCC countries are presented in this section, where the predictions take into consideration the proposed ANN framework, using MATLAB. K-frequency bands include Ku-Band 13-14 GHz, K-Band $18 \mathrm{GHz}$, and $\mathrm{Ka}$-Band $26.6 \mathrm{GHz}$, at CubeSat altitude of $400 \mathrm{~km}$ above the ground. These three types of variables in the framework of the proposed prediction can be classified into three groups as follows:

(i) Fixed parameters: these parameters are fixed input to the ANN, which are $P_{t}, G_{C}, G_{S}, d, \lambda, E_{r}, h_{\mathrm{t}}, F, K$, $T_{0}, B, B w$, and $R_{b}$

(ii) Variable parameters: these parameters are variable input to the ANN, which are $\theta, f$, longitude and latitude of the specific area in the GCC countries

(iii) Predicted parameters: these parameters are expected output from the ANN, which are $(C / N)_{T}$, $E_{b} / N_{0}, R_{A}, G_{A}, C_{A}, S_{A}, D_{A}$, and $E$ 


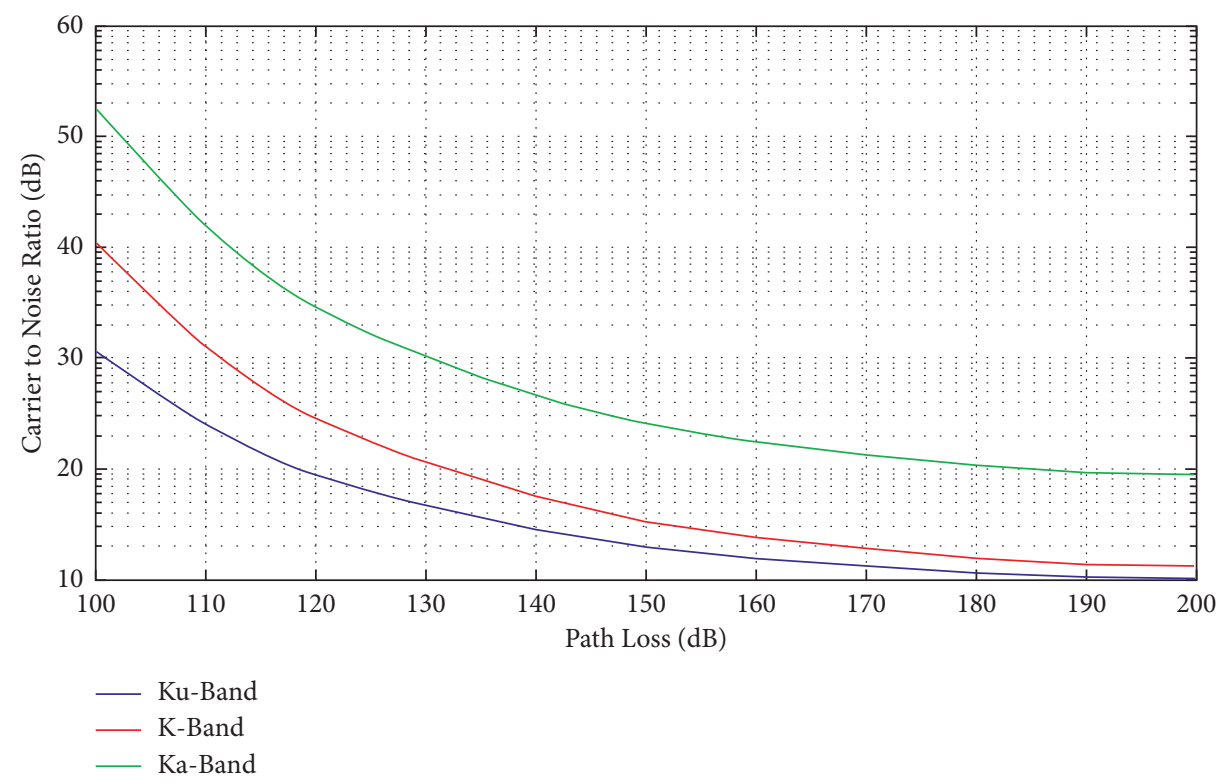

Figure 8: C/N as functions of path loss at different K-frequency bands.

Figures 8 and 9 visualize the link margin of the proposed CubeSat framework. Figure 8 shows $\mathrm{C} / \mathrm{N}$ as functions of path loss at different $\mathrm{K}$-frequency bands. When the frequency band increases, path loss increases too. Figure 9 reveals the BER of the signal as a function of $\mathrm{E} \_\mathrm{b} / \mathrm{N} \_\mathrm{O}$ at different $\mathrm{K}$-frequency bands. It is observed that an increase of the frequency offset increases the BER value at a fixed E_b/ N_o ratio. The best link performance is the one that allows for the lowest possible BER with the lowest possible E_b/ N_o. At the lowest BER achieved of $1 \times 10^{-6}$, the E_b/N_o with range floats between $13.5 \mathrm{~dB}$ and $16.5 \mathrm{~dB}$. It is observed that, as PL/frequency band increases, both BER and E_b/ N_o increase, and system performance drops gradually. Overall, these two performance quality indicators of the proposed CubeSat communication channel framework seem reasonable.

Figures 10-13 show the performance of the simulated MIMO antenna at K-frequency Bands. Figure 10 displays the specification and geometry of the $8 \times 8$ patch MIMO antenna configuration. Figure 11 presents an antenna gain where all patches are in-phase and beamforming has been steered at various angles $\left({ }^{\circ}\right)$ towards the desired direction. Figure 12 displays the return loss result S11 of the proposed feed geometry with respect to port. S11, which is also known as "reflection coefficient" represents how much power is reflected from the antenna. The $S$ parameter measures the resonant frequency of the antenna in relation to the resonance of the TM12 mode. Herein, it shows an accepted radiation power with low loss at 14,18 , and $26 \mathrm{GHz}$, respectively. Figure 13 demonstrates the Effective Isotropic Radiated Power (EIRP) of the proposed MIMO antenna to measure the coverage of the arrays, where it plots a Cumulative Distribution Function (CDF). Figure 13 shows that coverage just above $56 \%$ of the sphere has a positive gain at $23 \mathrm{dBmW}$ input power, representing an acceptable performance level.

Figures 14 and 15 show attenuations of rain, gasses, clouds, scintillation, and dust across wide range of frequency bands and elevation angles. Also, total attenuation, which includes rain, gasses, clouds, scintillation, and dust, of the CubeSat communication channel framework has been calculated. The average of the total attenuation at different frequency bands floats between 5 and $7 \mathrm{~dB}$. Clearly, dust attenuation registers the highest attenuation value compared to other attenuations. This is due to the physical and chemical characteristics of dust and sand particles (e.g., permittivity, size, concentration) that have an extra amount of radio wave energy dissipated by means of scattering and absorption. Thus, dust is the most vital cause of wireless satellite channel impairments, often observed in arid and semiarid areas such as GCC countries.

Another observation is that the attenuations increase as frequency increases along with a decrease in elevation angle. Unsurprisingly, high frequencies are more vulnerable to signal degradation, as radio signals get absorbed by atmospheric rain, snow, ice, or even dust, whereas the reason why attenuations increase positively with elevation angle is that distance increases accordingly at lower elevation angles. Path loss increases due to distance increases, whereas at high elevation angles, more LoS connectivity is achieved but less coverage footprint. Thus it is a trade-off.

Figures 16-19 depict performance results of the proposed ANN prediction framework. Batch training has been considered to train input sets via a learning algorithm in one epoch, which relates to the maximum iterations before weights get updated. Then, the process regulates the optimal number of iterations, during which validation produces a minimal value that can be visualized in regression plotting. Figure 16 shows the process of training the proposed ANN 


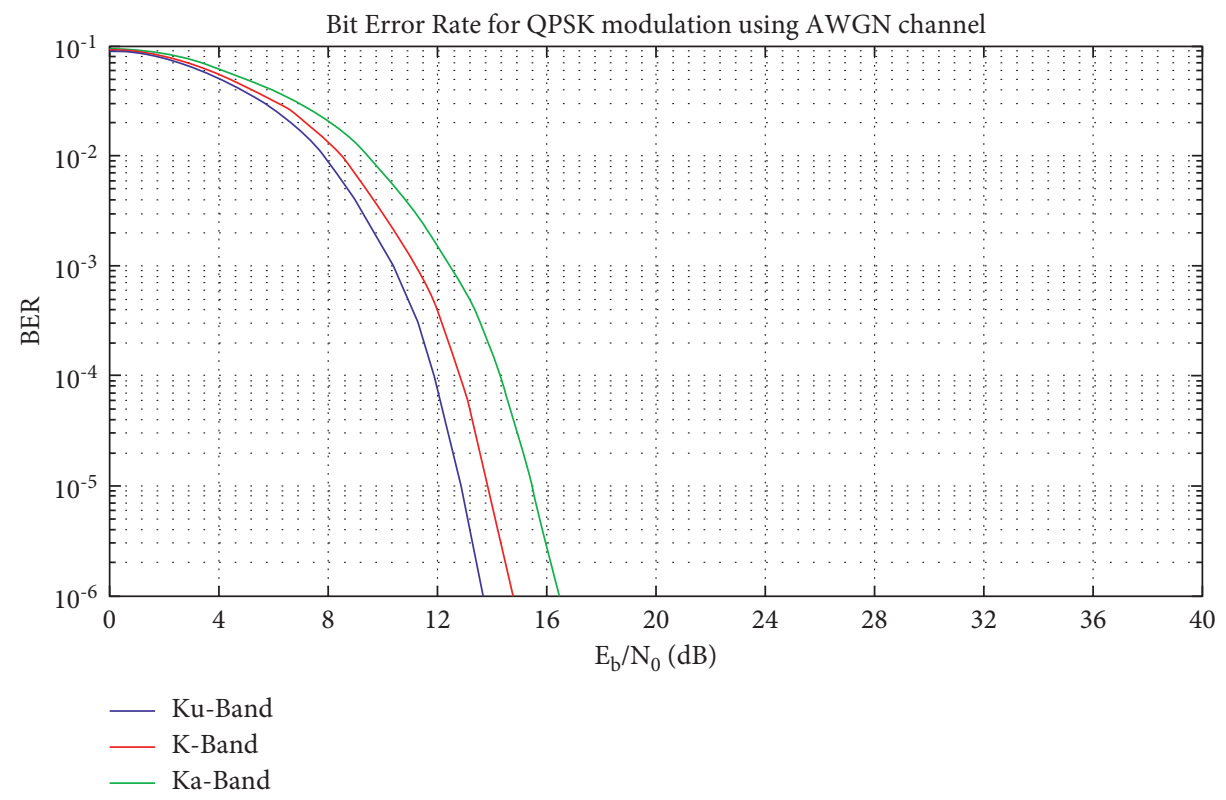

Figure 9: BER of the signal as a function of E_B/N_O at different K-frequency bands.

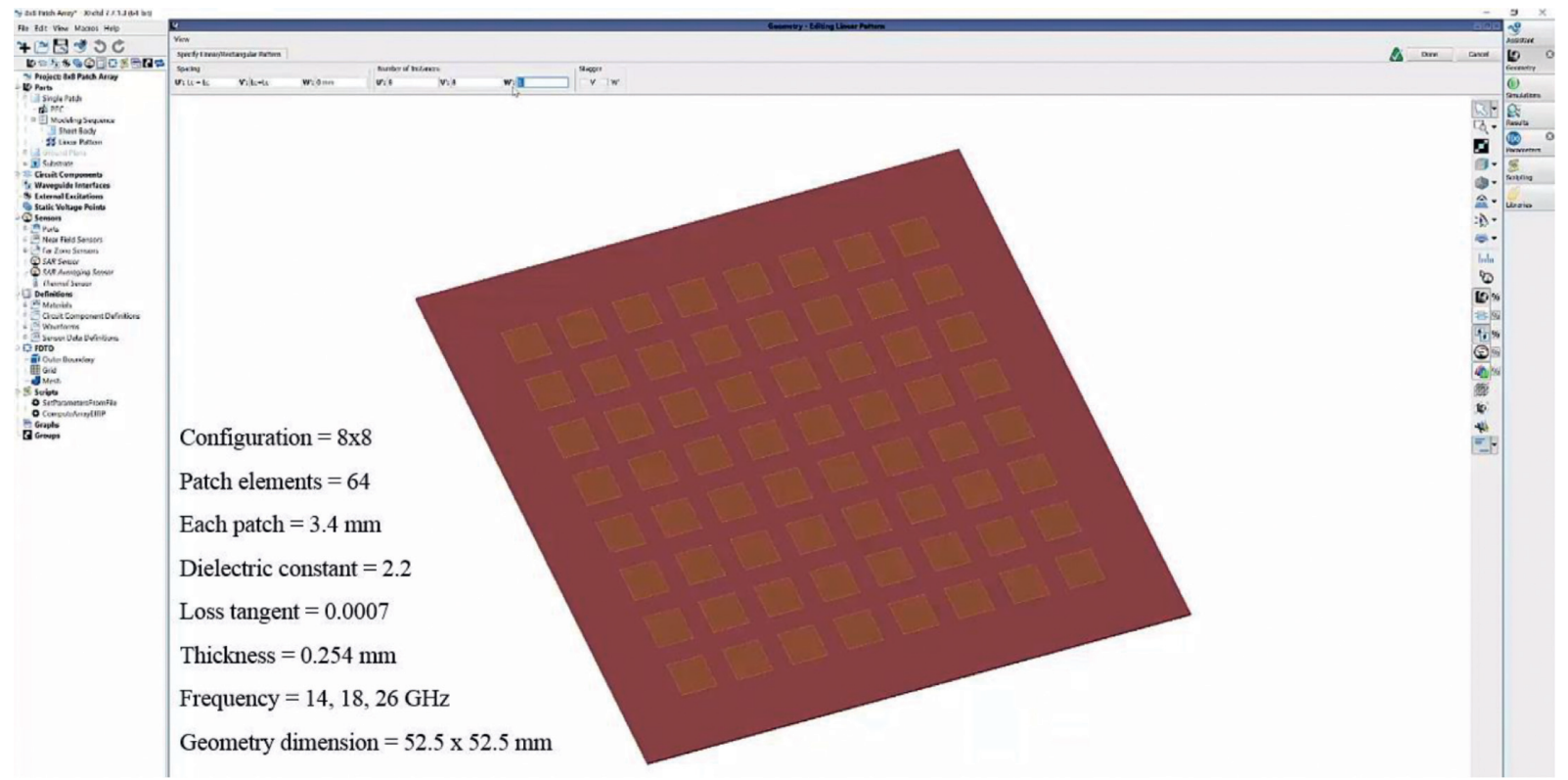

FIgURE 10: The antenna geometry and specification.

continues for 6 more iterations, during which error rates do not drop lower. However, in the $7^{\text {th }}$ iteration, training stops as the error rate increases. The result is reasonable due to three reasons: first, the concluding mean square error (MSE) is small; second, no substantial overfitting has occurred by iteration 7 , before which the best validation performance occurs; and third, the test set error and the validation set error have similar characteristics.

Figure 17 shows a 3-layer training performance of the proposed ANN prediction framework using Stochastic gradient descent (SGD), which refers to the value of the backpropagation gradient on each iteration in a logarithmic scale. So, at each iteration, the weights and biases get updated. Observing both values of gradient coefficient and $\mathrm{Mu}$ at their lowest minimum results indicate better training and testing of the ANN. However, validation checks represent the number of successive epochs in which the validation performance fails to decrease. Training stops when the validation parameter reaches the supreme number of validations of 6 at epoch 13 with the lowest gradient coefficient and $\mathrm{Mu}$ values. Overall performance shows that precise predictions may be obtained, where no 


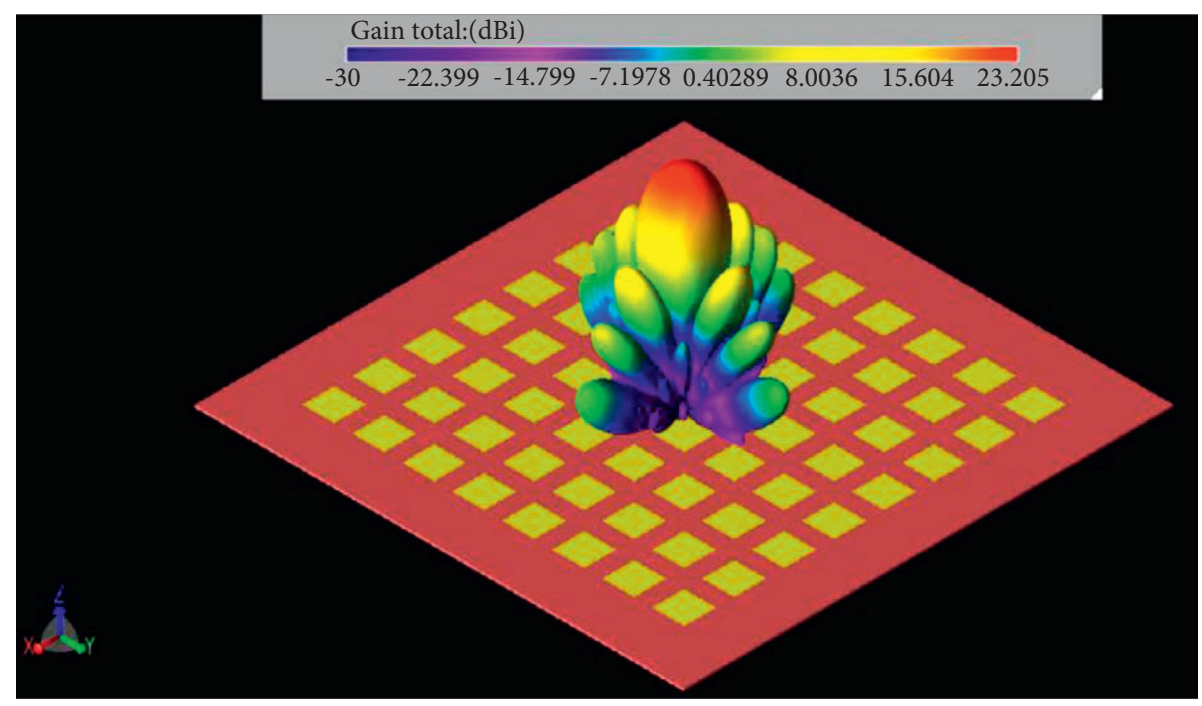

Figure 11: Antenna gain at various angles.

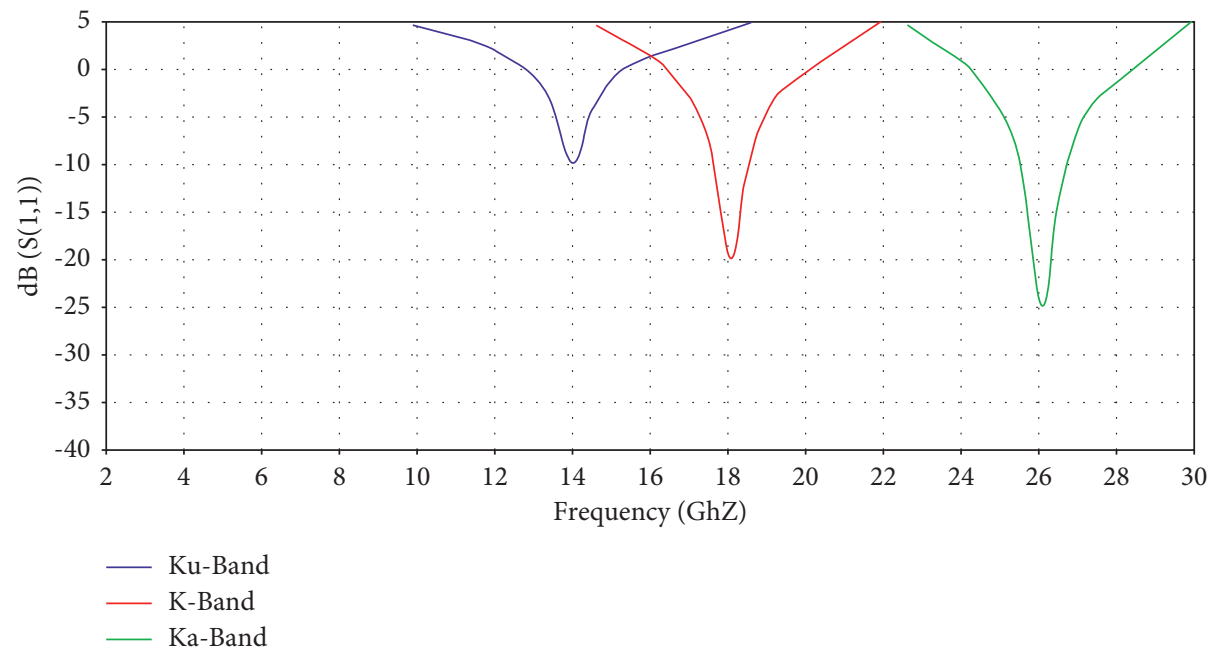

FIgURE 12: 5G MIMO antenna S11 results at K-frequency bands.

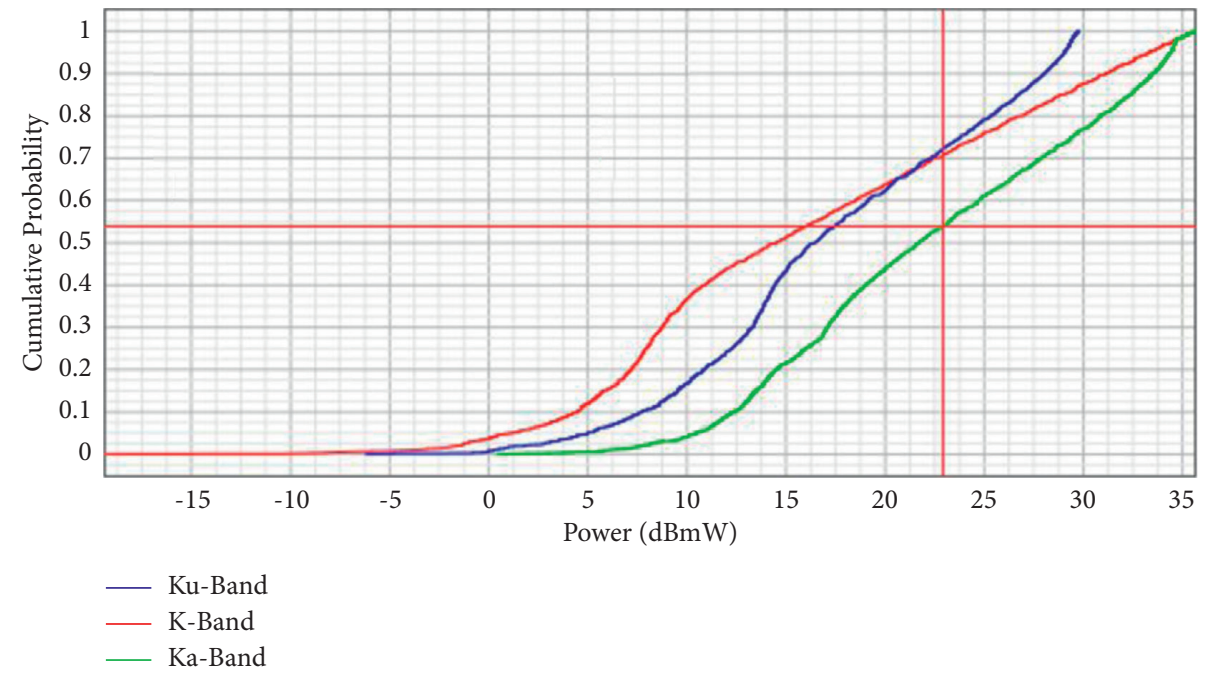

Figure 13: 5G MIMO antenna CDF of EIRP. 


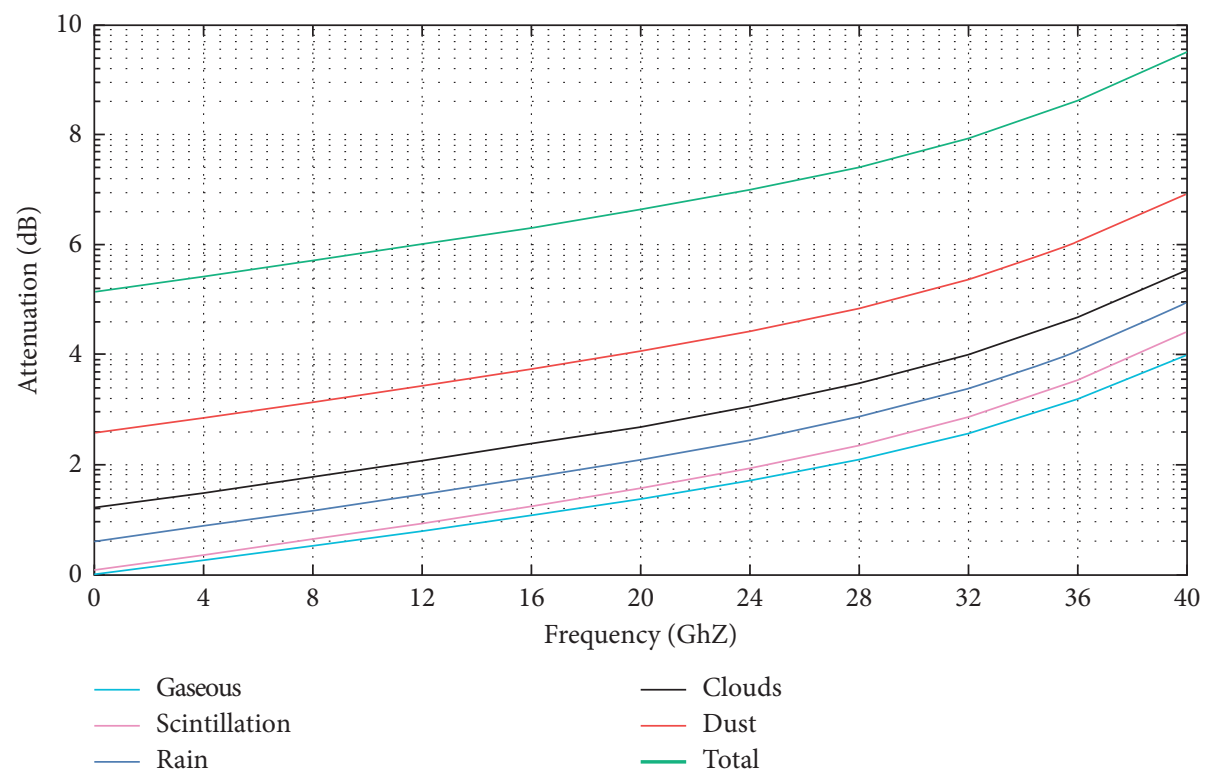

Figure 14: Different attenuations across a wide range of frequency bands.

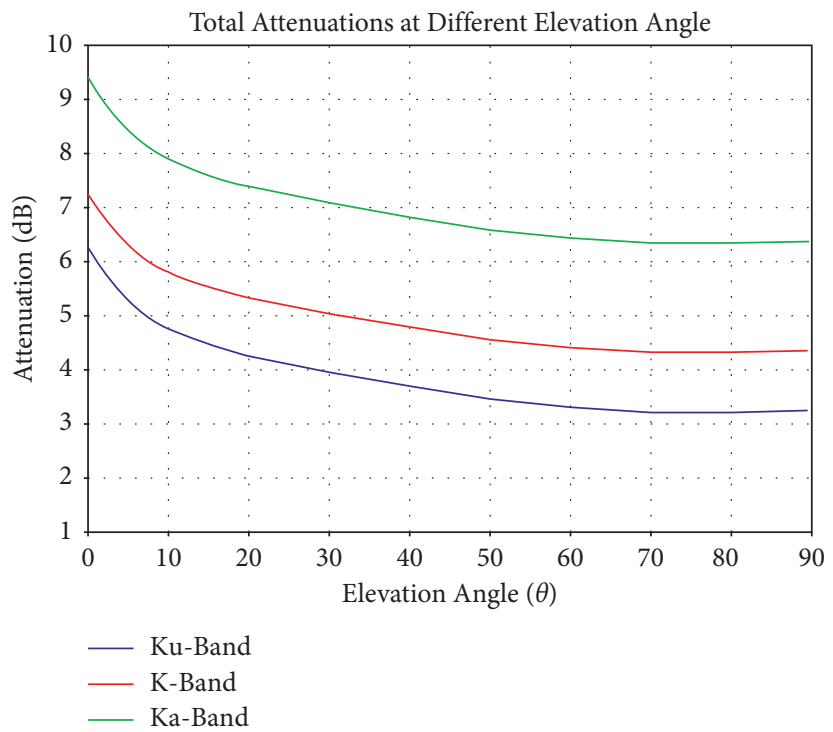

FIgURE 15: Total attenuations across K-frequency bands.

overfitting occurs before its best validation performance occurs.

Figure 18 demonstrates that regression plots target against training, validation, and test sets. Data must fall along a 45-degree line, where the outputs are equal to the targets to achieve a perfect fit. Targets mean the difference between the perfect result and the outputs. The dashed lines refer to the targets, while the solid lines refer to the best fit between targets and outputs. The $R$ value denotes the relation among the outputs and targets, where $R$ is equal or close to 1 . Then, there is a linear relation. Overall performance shows that $R$ values in the regression plots are satisfactory and represent the best fitness levels. Figure 19 displays the $E$ performance of attenuations prediction against the attenuations ANN prediction framework, where the proposed ANN approach is aimed at offering robust channel model planning, with full-range consideration of different attenuation impairments. The error performance of the proposed ANN approach shows lower value in comparison to nonoptimized predictions that describe a robust channel, where you can achieve low error rate without requiring a lot of transmission power. 


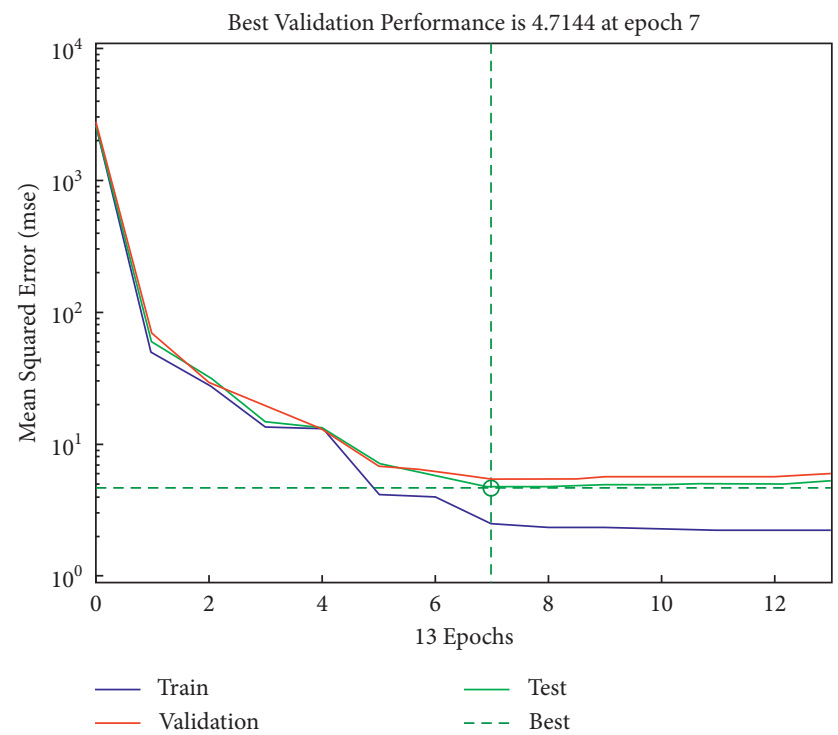

FIgURE 16: Training performance of the proposed ANN prediction framework.
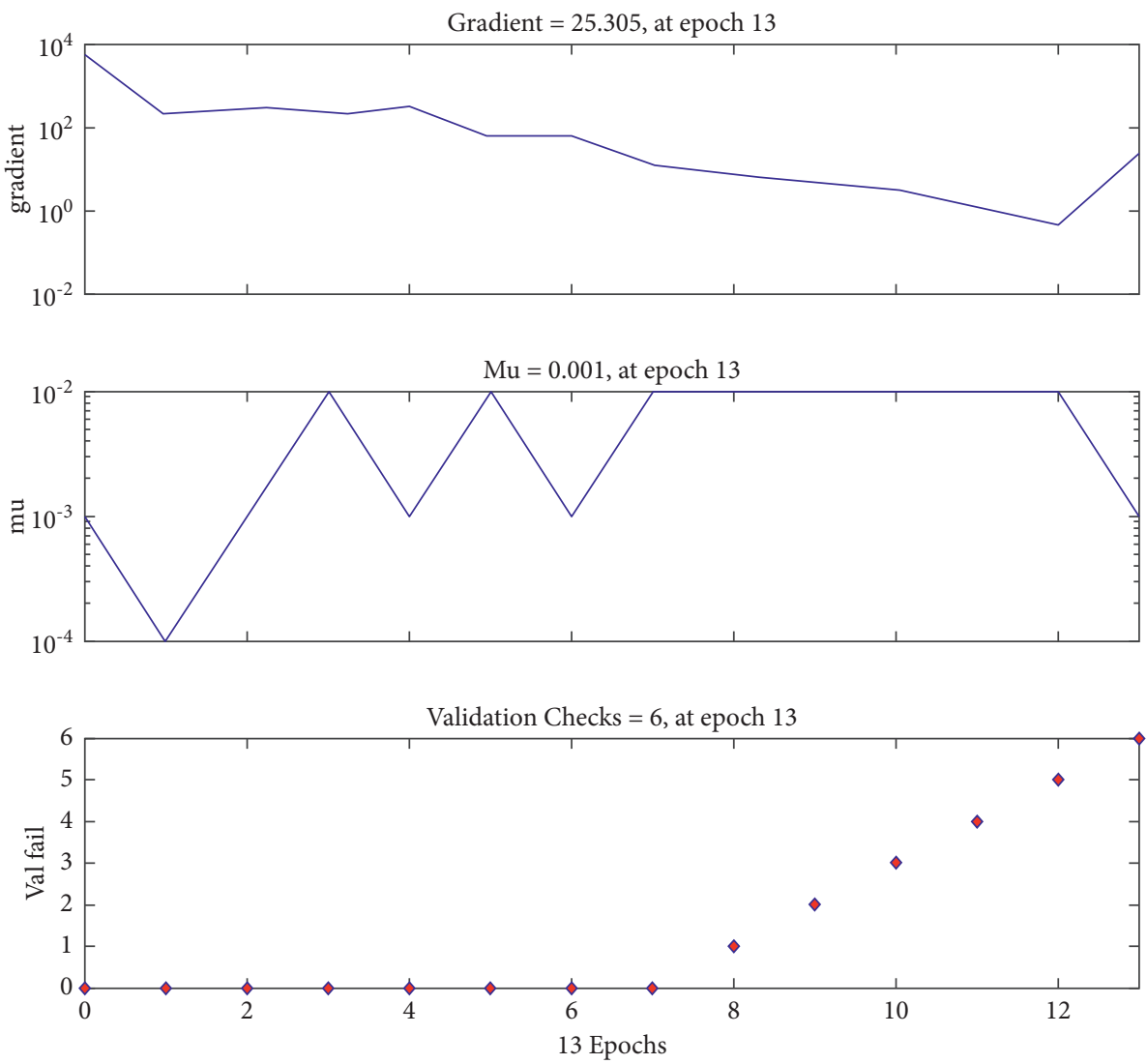

FIgURE 17: Training data of proposed ANN prediction framework using GSD. 

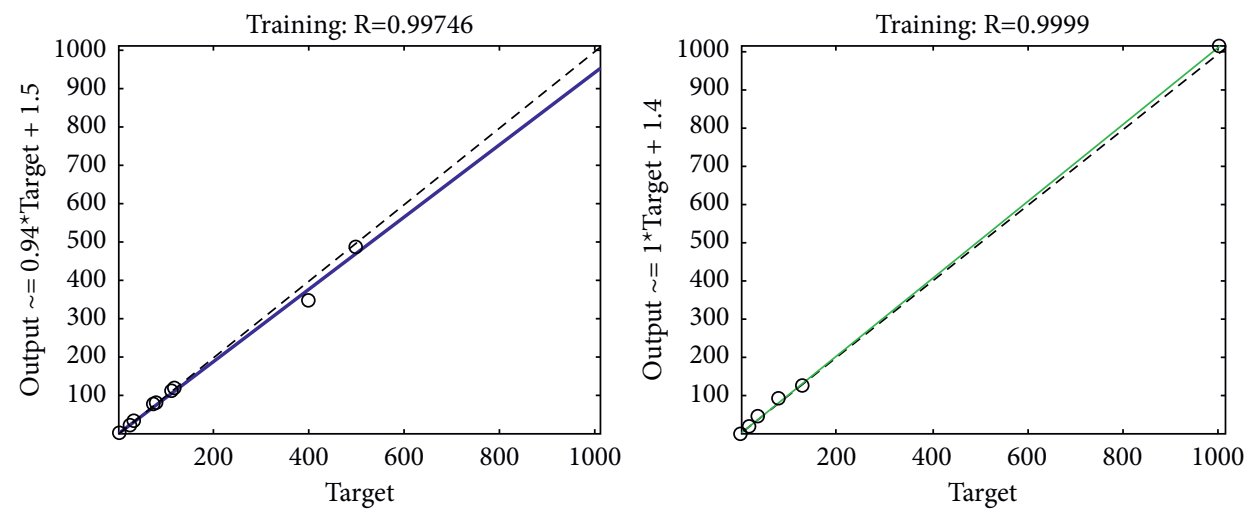

○ Data

- Fit

- - - $\mathrm{Y}=\mathrm{T}$

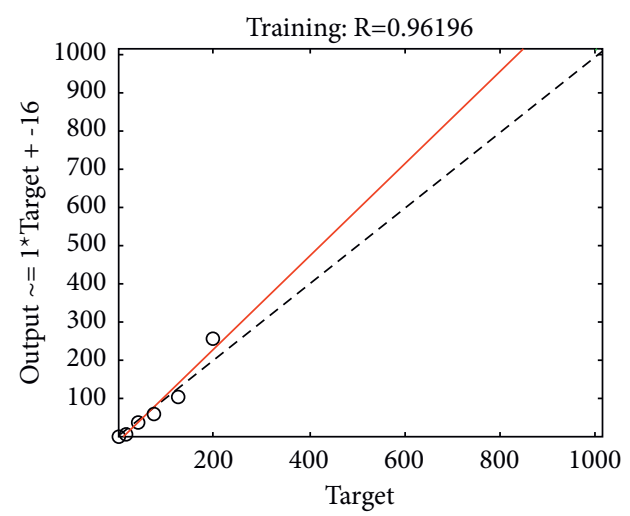

O Data

- Fit

- - $\mathrm{Y}=\mathrm{T}$

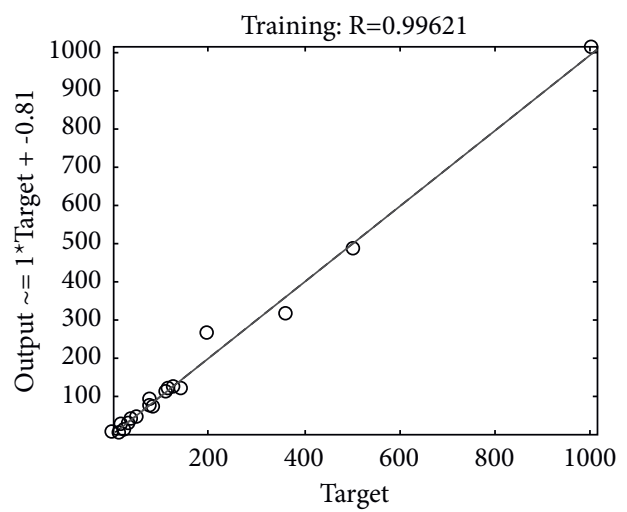

○ Data

O Data

- Fit

- Fit

$---\mathrm{Y}=\mathrm{T}$

$---\mathrm{Y}=\mathrm{T}$

FIGURE 18: Regression plots of the ANN prediction framework.

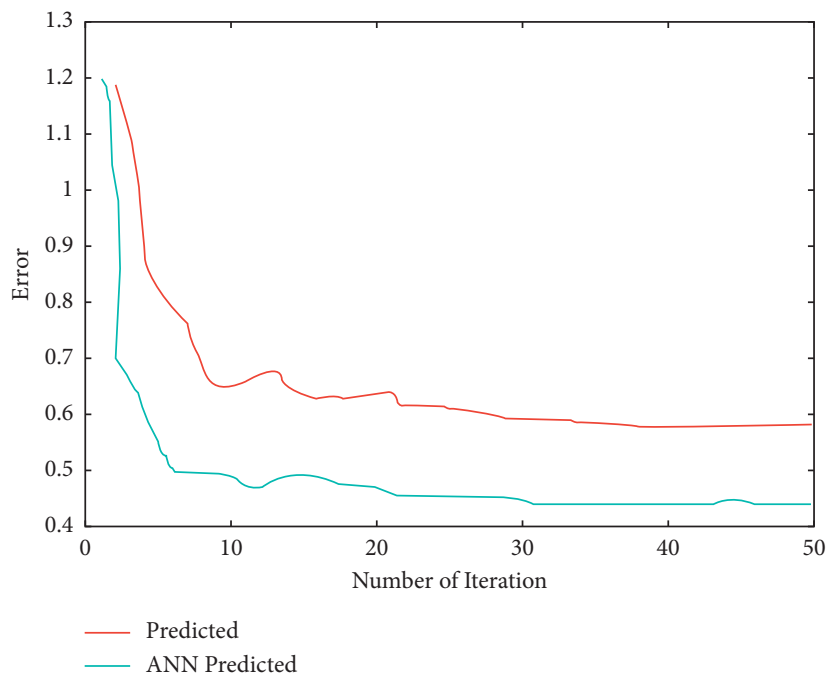

FIGURE 19: E performance of attenuations prediction against the attenuations ANN prediction framework.

\section{Conclusion}

Providing seamless communication services using spacebased systems, such as micro-/nanosatellites (e.g., CubeSat), would massively pave the way to hyperconnected societies and thus more global smart connectivity. Still, atmospheric and dust impairments are open challenges that face satellite systems, especially at high-frequency bands in arid and 
semiarid regions. Motivated by this, this paper aims to provide a comprehensive prediction model that encompasses joint effects of atmospheric and dust impairments from CubeSat communication aspect in GCC) region using ANN framework for CubeSat communication. The prediction model also considers a MIMO antenna to obtain better QoS and capacity. The predicted results confirm that the comprehensive prediction model for CubeSat using ANN offers much promise channel model for satellite communications, which in turn leads to not only supporting IoT connectivity but also reducing power consumption, thus enhancing lifetime of CubeSat. Further, the overall ANN model outperforms the nonoptimized model. As future work, generating predictions with fine-tune environmental considerations in any location using a model that integrates Google Maps is gaining momentum. Further, this work has considered OFDM technology with the proposed massive MIMO antenna. Nevertheless, Filter Bank Multicarrier (FBMC) can be investigated further as proposed in [57-59] but from spec-based systems such as CubeSat perspectives because of its efficiency of high date rate and low latency.

\section{Data Availability}

The data used to support the findings of this study are included within the article.

\section{Conflicts of Interest}

The authors declare no conflicts of interest.

\section{Acknowledgments}

This research was funded by the Deanship of Scientific Research at Taif University, Kingdom of Saudi Arabia, Taif University Researchers Supporting Project (no. TURSP2020/265).

\section{References}

[1] N. Saeed, A. Elzanaty, H. Almorad, H. Dahrouj, T. Y. AlNaffouri, and M.-S. Alouini, "CubeSat communications: recent advances and future challenges," IEEE Communications Surveys \& Tutorials, vol. 22, no. 3, pp. 1839-1862, 2020, thirdquarter 2020.

[2] C. Nieto-Peroy and M. R. Emami, "CubeSat mission: from design to operation," Applied Sciences, vol. 9, no. 15, p. 3110, 2019.

[3] F. A. Almalki, "Utilizing drone for food quality and safety detection using wireless sensors," in Proceedings of the 2020 IEEE 3rd International Conference on Information Communication and Signal Processing (ICICSP), pp. 405-412, Shanghai, China, 2020.

[4] F. Almalki, "Developing an adaptive channel modelling using a genetic algorithm technique to enhance aerial vehicle-toeverything wireless communications," International Journal of Computer Networks \& Communications, vol. 13, no. 2, pp. 37-56, 2021, Available:.

[5] J. W. Cheong, B. J. Southwell, W. Andrew et al., "A robust framework for low-cost CubeSat scientific missions," Space Science Reviews, vol. 216, no. 1, 2020.
[6] X. Fang, W. Feng, T. Wei, Y. Chen, N. Ge, and C.-X. Wang, " $5 \mathrm{G}$ embraces satellites for $6 \mathrm{G}$ ubiquitous IoT: basic models for integrated satellite terrestrial networks," IEEE Internet of Things Journal, vol. 8, no. 18, pp. 14399-14417, 2021.

[7] S. H. Alsamhi, F. A. Almalki, H. Al-Dois et al., "Machine learning for smart environments in B5G networks: connectivity and QoS," Computational Intelligence and Neuroscience, vol. 2021, Article ID 6805151, 23 pages, 2021.

[8] J. Junior, A. Ambrosio, and F. Sousa, "Real-time cubesat thermal simulation using artificial neural networks," Journal of Computational Interdisciplinary Sciences, vol. 8, no. 2, 2017.

[9] F. A. Almalki, A. A. Alotaibi, and M. C. Angelides, "Coupling multifunction drones with AI in the fight against the coronavirus pandemic," Computing, vol. 103, no. 11, 2021.

[10] G. Villarrubia, J. F. De Paz, P. Chamoso, and F. D. la Prieta, "Artificial neural networks used in optimization problems," Neurocomputing, vol. 272, pp. 10-16, 2018.

[11] J. E. Rayas-Sanchez, "EM-based optimization of microwave circuits using artificial neural networks: the state-of-the-art," IEEE Transactions on Microwave Theory and Techniques, vol. 52, no. 1, pp. 420-435, 2004.

[12] S. H. Alsamhi, O. Ma, M. S. Ansari, and F. A. Almalki, "Survey on collaborative smart drones and internet of Things for improving smartness of smart cities," IEEE Access, vol. 7, pp. 128125-128152, 2019.

[13] F. A. Almalki, B. O. Soufiene, S. H. Alsamhi, and H. Sakli, “A low-cost platform for environmental smart farming monitoring system based on IoT and UAVs," Sustainability, vol. 13, no. 11, p. 5908, 2021.

[14] F. A. Almalki, S. H. Alsamhi, R. Sahal et al., "Green IoT for eco-friendly and sustainable smart cities: future directions and opportunities," Mobile Networks and Applications, vol. 26, no. $4,2021$.

[15] F. A. Almalki, S. Ben Othman, F. A. Almalki, H. Sakli, and EERP-DPM, "Energy efficient routing protocol using dual prediction model for healthcare using IoT," Journal of Healthcare Engineering, vol. 2021, Article ID 9988038, 15 pages, 2021.

[16] A. Camps, "Nanosatellites and Applications to Commercial and Scientific Missions Satellites Missions And Technologies For Geosciences, pp. 1-25, Intechopen, London, UK, 2019.

[17] F. A. Almalki and M. C. Angelides, "Considering near space platforms to close the coverage gap in wireless communications: the case of the Kingdom of Saudi Arabia," in Proceedings of the 2016 Future Technologies Conference (FTC), pp. 224-230, San Francisco, USA, 2016.

[18] Canadian Space Agency, "What is a CubeSat," 2017, http://www.asc-csa.gc.ca.

[19] A. Kak and I. F. Akyildiz, "Designing large-scale constellations for the internet of space Things with CubeSats," IEEE Internet of Things Journal, vol. 8, no. 3, pp. 1749-1768, 2021.

[20] F. Almalki, "Comparative and QoS performance analysis of terrestrial-aerial platforms-satellites systems for temporary events," International journal of Computer Networks \& Communications, vol. 11, no. 6, pp. 111-133, 2019.

[21] R. Sinha, A. Verma, and S. Choudhary, "General analysis of cube satellite technology: an overview," International Journal of Computer Application, vol. 178, no. 21, pp. 19-22, 2019.

[22] F. A. Almalki and M. C. Angelides, "Empirical evolution of a propagation model for low altitude platforms," in Proceedings of the 2017 Computing Conference, pp. 1297-1304, London, UK, 2017.

[23] M. M. Nair, A. K. Tyagi, and N. Sreenath, "The future with industry 4.0 at the core of society 5.0: open issues, future 
opportunities and challenges," in Proceedings of the 2021 International Conference on Computer Communication and Informatics (ICCCI), pp. 1-7, Coimbatore, India, 2021.

[24] P. Machuca, J. P. Sánchez, and S. Greenland, “Asteroid flyby opportunities using semi-autonomous CubeSats: mission design and science opportunities," Planetary and Space Science, vol. 165, pp. 179-193, 2019.

[25] I. F. Akyildiz and A. Kak, "The internet of space things/ CubeSats," IEEE Network, vol. 33, no. 5, pp. 212-218, 2019.

[26] Spaceworks Enterprises Inc, "Nano/microsatellite market forecast," 2019, https://www.spaceworks.aero/wp-content/ uploads/Nano-Microsatellite-Market-Forecast-9th-Edition2019.pdf.

[27] K. Woellert, P. Ehrenfreund, A. J. Ricco, and H. Hertzfeld, "CubeSats: cost-effective science and technology platforms for emerging and developing nations," Advances in Space Research, vol. 47, no. 4, pp. 663-684, 2011.

[28] A. R. Aslan, H. M. K. AlNaimiy, I. Fernini et al., "Space technology capacity building in support of SDG 2030 through CubeSat SharjahSat-1," in Proceedings of the 2019 9th International Conference on Recent Advances in Space Technologies (RAST), pp. 955-958, Istanbul, Turkiye, 2019.

[29] I. F. Akyildiz, J. M. Jornet, and S. Nie, “A new CubeSat design with reconfigurable multi-band radios for dynamic spectrum satellite communication networks," Ad Hoc Networks, vol. 86, pp. 166-178, 2019.

[30] Z. Qu, G. Zhang, H. Cao, and J. Xie, "Stochastic dynamic modeling of rain attenuation: a survey," China Communications, vol. 15, no. 3, pp. 220-235, 2018.

[31] L. Luini and C. Capsoni, "Joint effects of clouds and rain on ka-band earth observation data downlink systems," in Proceedings of the 9th European Conference on Antennas and Propagation (EuCAP), pp. 1-5, Lisbon, Portugal, 2015.

[32] L. J. Mpoporo and P. A. Owolawi, "Comparison of measured and estimated rain attenuation on earth-satellite links in South Africa," in Proceedings of the 2019 International Multidisciplinary Information Technology and Engineering Conference (IMITEC), pp. 1-5, Kimberley, South Africa, 2019.

[33] J. Wang, L. Wang, and M. Xu, "Rain attenuation analysis of Ka band ship-borne satellite communication station in Indian Ocean and pacific ocean," in Proceedings of the 2020 IEEE 3rd International Conference on Information Communication and Signal Processing (ICICSP), pp. 385-388, Shanghai, China, 2020.

[34] M. R. Averly and J. Suryana, "CubeSat communication system for maritime needs," in Proceedings of the 2020 27th International Conference on Telecommunications (ICT), pp. 1-5, Shanghai, China, 2020.

[35] E. Regonesi, L. Luini, and C. Riva, "Limitations of the ITU-R P.838-3 model for rain specific attenuation," in Proceedings of the 13th European Conference on Antennas and Propagation (EuCAP), pp. 1-4, Krakow, Poland, 2019.

[36] A. G. Cappiello, D. C. Popescu, J. S. Harris, and O. Popescu, "Radio link design for CubeSat-to-ground station communications using an experimental license," in Proceedings of the 2019 International Symposium on Signals, Circuits and Systems (ISSCS), pp. 1-4, Iasi, Romania, 2019.

[37] G. Charbit, D. Lin, K. Medles, L. Li, and I.-K. Fu, "Spaceterrestrial radio network integration for IoT," in Proceedings of the 2020 2nd 6G Wireless Summit (6G SUMMIT), pp. 1-5, Levi, Finland, 2020.

[38] K. Harb, O. Butt, A. A. Al-Yami, and S. Abdul-Jauwad, "Probabilistic dust storm layers impacting satellite communications," in Proceedings of the 2013 IEEE International
Conference on Space Science and Communication (IconSpace), pp. 407-411, Melaka, Malaysia, 2013.

[39] K. Harb, B. Omair, S. Abdul-Jauwad, A. Al-Yami, and A. AlYami, "A Proposed Method for Dust and Sand Storms Effect on Satellite Communication Networks, Innovations On Communication Theory, Istanbul, Turkey, 2012.

[40] F. A. Almalki and M. C. Angelides, "Propagation modelling and performance assessment of aerial platforms deployed during emergencies," in Proceedings of the 2017 12th International Conference for Internet Technology and Secured Transactions (ICITST), pp. 238-243, Cambridge, UK, 2017.

[41] D. Barbaric, J. Vukovic, and D. Babic, "Link budget analysis for a proposed CubeSat Earth observation mission," in Proceedings of the 2018 41st International Convention on Information and Communication Technology, Electronics and Microelectronics (MIPRO), pp. 0133-0138, Cambridge, UK, 2018.

[42] S. Chen, J. Zhang, Y. Jin, and B. Ai, "Wireless powered IoE for 6G: massive access meets scalable cell-free massive MIMO," China Communications, vol. 17, no. 12, pp. 92-109, 2020.

[43] F. A. Almalki and M. C. Angelides, "An enhanced design of a 5G MIMO antenna for fixed wireless aerial access," Cluster Computing, vol. 24, no. 3, 2021.

[44] S. Abulgasem, F. Tubbal, R. Raad, P. I. Theoharis, S. Lu, and S. Iranmanesh, "Antenna designs for CubeSats: a review," IEEE Access, vol. 9, pp. 45289-45324, 2021.

[45] D. Mansukhani, "Satellite communication: signal attenuation by rain and clouds," International Journal of Electrical, Electronics And Data Communication, vol. 5, no. 11, pp. 3638, 2017.

[46] N. Aida M.Nor, M. R. Islam, W. Al-Khateeb, and S. A.Z, "Atmospheric effects on free space earth-to-satellite optical link in tropical climate," International Journal of Computer Science, Engineering and Applications, vol. 3, no. 1, pp. 17-36, 2013.

[47] A. Mansourkhaki, M. Berangi, and M. Haghiri, "Comparative application of radial basis function and multilayer perceptron neural networks to predict traffic noise pollution in tehran roads," Journal of Ecological Engineering, vol. 19, no. 1, pp. 113-121, 2018.

[48] F. A. Almalki and M. C. Angelides, "Deployment of an aerial platform system for rapid restoration of communications links after a disaster: a machine learning approach," Computing, vol. 102, no. 4, pp. 829-864, 2019.

[49] W. Jiang and H. D. Schotten, "Neural network-based fading channel prediction: a comprehensive overview," IEEE Access, vol. 7, pp. 118112-118124, 2019.

[50] Y. Yanqin Zuo, F. Chengrui Jia, and C. Jia, "Prediction for satellite communication based on data mining," in Proceedings of the 15th International Conference on Optical Communications and Networks (ICOCN), pp. 1-3, 2016.

[51] F. A. Almalki and Ben Othman Soufiene, "EPPDA: an efficient and privacy-preserving data aggregation scheme with authentication and authorization for IoT-based healthcare applications," Wireless Communications and Mobile Computing, vol. 2021, Article ID 5594159, 18 pages, 2021.

[52] A. O. Ayo, P. A. Owolawi, J. S. Ojo, and L. J. Mpoporo, "Rain impairment model for satellite communication link design in South Africa using neural network," in Proceedings of the 2nd International Multidisciplinary Information Technology and Engineering Conference (IMITEC), pp. 1-8, Kimberley, South Africa, 2020.

[53] F. A. Almalki and M. C. Angelides, "A machine learning approach to evolving an optimal propagation model for last 
mile connectivity using low altitude platforms," Computer Communications, vol. 143, pp. 9-33, 2019.

[54] P. Thiennviboon and S. Wisutimateekorn, "Rain attenuation prediction modeling for earth-space links using artificial neural networks," in Proceedings of the 2019 16th International Conference on Electrical Engineering/Electronics, Computer, Telecommunications and Information Technology (ECTICON), pp. 29-32, 2019.

[55] S. H. Alsamhi, F. Almalki, O. Ma, M. S. Ansari, and B. Lee, "Predictive estimation of optimal signal strength from drones over IoT frameworks in smart cities," IEEE Transactions on Mobile Computing, vol. 2021, Article ID 6805151, 1 page, 2021.

[56] F. Fourati and M.-S. Alouini, "Artificial intelligence for satellite communication: a review," 2021, https://arxiv.org/abs/ 2101.10899.

[57] D. Kong, X. Zheng, Y. Zhang, and T. Jiang, "Frame repetition: a solution to imaginary interference cancellation in FBMC/ OQAM systems," IEEE Transactions on Signal Processing, vol. 68, pp. 1259-1273, 2020.

[58] H. Wang, X. Li, R. H. Jhaveri et al., "Sparse Bayesian learning based channel estimation in FBMC/OQAM industrial IoT networks," Computer Communications, vol. 176, pp. 40-45, 2021.

[59] D. Kong, J. Li, K. Luo, and T. Jiang, "Reducing pilot overhead: channel estimation with symbol repetition in MIMO-FBMC systems," IEEE Transactions on Communications, vol. 68, no. 12, pp. 7634-7646, 2020. 